\title{
Models for radioactivity dispersion assessments in Andalusian coastal waters: Gulf of Cádiz and Alborán Sea
}

\author{
R. Periáñez \\ Department of Applied Physics 1, EUITA, Universidad de Sevilla, Ctra Utrera km 1, \\ 41013-Sevilla, Spain
}

\begin{abstract}
Rapid response models for the assessment of the consequences of a radioactive spill in the coastal waters of Andalusia (south of Spain) have been developed. These waters comprise the Gulf of Cadiz, GoC, (Atlantic Ocean) and the Alborán Sea, AS, (Mediterranean Sea) and two models are described, covering both sectors. This subject is of high relevance since these waters are the only connection between the Atlantic and the Mediterranean. Thus, there are intense shipping activities that for instance include the transit of nuclear submarines. The models include hydrodynamic sub-models appropriate to describe the water circulation in each area. Results from the hydrodynamic models have been carefully tested through comparisons with observed tides and currents. The dispersion models are based upon a particle-tracking technique. Thus, the radioactive spill is simulated by a number of discrete particles, each one equivalent to a number of units, whose paths are computed. Turbulent diffusion and radioactive decay are calculated using a stochastic Monte Carlo method. The radionuclide concentrations may be obtained at the desired time from the density of particles per water volume. Some applications and examples of results are given.
\end{abstract}

\section{INTRODUCTION}

Numerical models for simulating pollutant dispersion are nowadays being developed since they can be used for decision-making purposes after releases of contaminants into the marine environment. In particular, particle-tracking methods are well suited for problems in which high contamination gradients are involved, since they do not introduce numerical diffusion. Also, they can give very fast answers, specially if the hydrodynamic calculations are made off-line and tidal analysis and computed residuals are used to reconstruct water movements, which avoids the CFL (Courant-Friederichs-Lewy) stability limitations in the dispersion calculations. Thus, particle-tracking models are very useful predictive tools that can be used for assessing contamination after accidental or deliberate releases of radioactivity in the marine environment.

The area of the GoC and AS has a high ecological value, being essential in marine and aerial migratory processes. As the only connection between the Mediterranean Sea and the Atlantic Ocean, marine turtles and mammals travel through the Strait of Gibraltar. Also, the region has a high tourist interest, since many $\mathrm{km}$ of sand beaches attract thousands of tourists each year. Finally, there are also some important towns. Shipping activities in the area of interest are very intense, again due to the fact that it is the only connection between the Atlantic and the Mediterranean, and the transit of nuclear submarines must be considered. A release of radioactivity in the area as a consequence of an accident (or a deliberate release) can lead to relevant ecological and economic impacts.

The objective of this work consists of describing two rapid-response models developed to simulate the dispersion of radioactive spills occurring in the GoC and the AS. The hydrodynamic is solved in advance. A 2D depth-averaged barotropic model is used to obtain tidal currents in both regions. The residual (mean) circulation is obtained from a 2-layer model and a full 3D baroclinic model in the AS and $\mathrm{GoC}$ respectively. Tidal and mean circulation are stored in files that will be read by the dispersion codes to compute water current at any time and position. Dispersion is solved using a particle tracking method. Thus, the spill is simulated by a number of particles, each of them equivalent to a number 
of units (for instance $\mathrm{Bq}$ ), whose paths are followed in time. Radioactive decay is included in the model using a stochastic technique. A Monte Carlo random-walk method is used to calculate turbulent diffusion. Contaminant concentrations may be obtained at the desired time from the density of particles per water volume unit. The models are briefly described in the following section, next some examples of results are presented.

\section{MODEL DESCRIPTIONS}

Currents at any given time and position, which are required to solve the advective transport of radionuclides, are obtained through the addition of the instantaneous tidal current plus the residual flow. Indeed, tides are relevant for the transport of pollutants in the area close to the Strait of Gibraltar, where associated currents are stronger. Inside the AS and the GoC, where weaker tidal currents exist, residual circulation is dominant. The spatial domain of the models may be seen in Fig. 1. Spatial resolution of the hydrodynamic models is 2 minutes in both longitude and latitude.

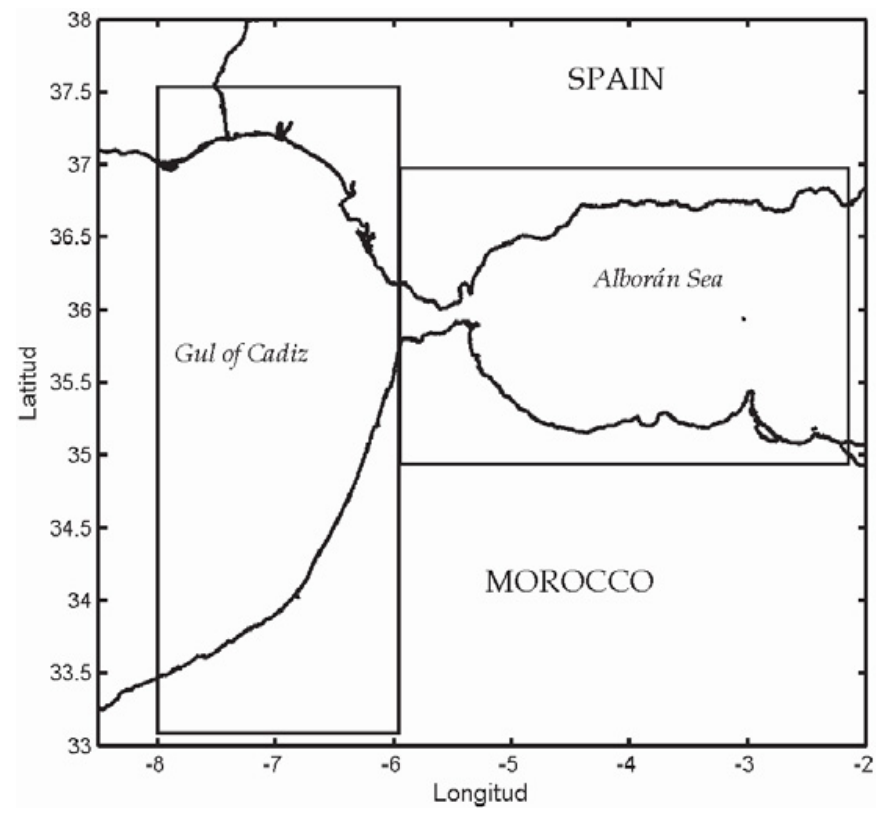

Figure 1. Spatial domain of the models covering the GoC and the AS (rectangular boxes).

Tides are calculated using a 2D-depth averaged model in each domain, which is a reasonable approach [1]. The two main constituents, $\mathbf{M}_{2}$ and $\mathrm{S}_{2}$ are included. Tidal analysis is carried out and tidal constants are stored in files that will be read by the dispersion code. This method, standard in rapid-response models, allows a fast determination of tidal currents at any time and position [1]. The 2D depth-averaged hydrodynamic equations are standard and may be seen elsewhere [2]. They are solved by means of explicit finite difference schemes with appropriate boundary conditions in each domain.

A 2-layer model is applied to obtain the residual circulation in the AS. This is a reasonable approach [3] since circulation in the AS may be seen as a surface flow of Atlantic Water moving to the east and a deep flow of more dense Mediterranean Water moving to the west. The main feature of circulation in the surface layer is an almost permanent anticyclonic gyre which fills most of the western AS known as the WAG (Western Alboran Gyre) [3]. The outflow of Mediterranean Water to the Atlantic Ocean mostly occurs along the Moroccan continental slope. Equations are solved using finite differences. Boundary 
conditions consist of the mean water flows through the Strait of Gibraltar [3]. Hydrodynamic equations may be seen in this reference as well.

A full 3D baroclinic model [4] has been used to obtain the residual circulation in the GoC since more water masses with different properties (temperature and salinity) are present here. The model includes equations for the evolution of salinity and temperature, and water density is obtained from them through a standard equation of state. The vertical eddy viscosity is obtained from a 1-equation turbulence model. Again finite differences are applied to integrate the equations and open boundary conditions are obtained from the WOA05 (World Ocean Atlas, 2005), available on-line from the NOAA (National Ocean and Atmosphere Administration, US).

Residual circulations in both domains are stored in files which will be read by the dispersion code. Current at any time and position is obtained through the addition of the instantaneous tidal current and the residual (mean) current at such position. The dispersion model formulation is the same in both the GoC and AS.

A radioactive spill is simulated as a discrete number of particles, each one equivalent to a number of units. Advection is computed from an equation in the form [1, 2]:

$$
\frac{\vec{r}(t+\Delta t)-\vec{r}(t)}{\Delta t}=\vec{q}(t)
$$

where $r$ is the position vector of the particle and $q$ is the total current (tidal plus residual) vector at the particle position. Three dimensional diffusion is computed using a random-walk procedure and radioactive decay is treated using a stochastic method [1], which is standard in particle-tracking models. The maximum sizes of the horizontal and vertical steps given by each particle due to turbulent diffusion are [2]:

$$
D_{h}=\sqrt{12 K_{h} \Delta t} \quad D_{v}=\sqrt{2 K_{v} \Delta t}
$$

where $K_{h}$ and $K_{v}$ are, respectively, horizontal and vertical diffusion coefficients and $\Delta t$ is time step in the dispersion calculations. The effect of wind is included as usual in particle-tracking models. Thus, it is assumed that the water surface moves in the direction of wind at a speed equal to $3 \%$ of the wind speed $10 \mathrm{~m}$ above the sea surface. This current decreases logarithmically to zero at a depth usually taken as 20 $\mathrm{m}$. Variable winds may be used. Date and time of the discharge (and duration in the case of continuous releases) must be specified since the fate of the release will depend on the tidal state when it took place. Thus, the appropriate phase of each tidal constituent at $t=0$ must be specified. The values used in both models correspond to the origin of time being January 1, 2003 at 0:15 hours Greenwich time. A spill occurring at any depth below the surface can be simulated. In their present formulations, the models neglect the interactions of dissolved radionuclides with sediments.

Twelve snapshots at constant intervals during the simulation may be plotted to show the evolution of the radionuclide patch over time. Final radionuclide concentrations at any depth over the domain computed from the density of particles per water volume unit are also provided. More details about the practical aspects of computation may be seen, for instance, in [2].

\section{RESULTS}

As an example, the computed residual circulation in the GoC is presented in Fig. 2. Currents at the surface and at $190 \mathrm{~m}$ depth are shown for the northern GoC. The residual surface circulation in the $\mathrm{GoC}$ is characterized by a current directed to the southeast along the Spanish coast. This circulation is a rather constant pattern during most of the year. Part of the flow enters the Strait of Gibraltar and part is deflected to the south. Below, the Mediterranean waters, coming into the Atlantic, flow to the northwest. The magnitude of the surface residual current is of the order of $10 \mathrm{~cm} / \mathrm{s}$, increasing as approaching the entrance of the Strait of Gibraltar. Additional validation of the 3D baroclinic model for the GoC has 
been obtained through the comparison of computed and measured vertical profiles of water temperature and salinity at several points (results not shown).

In the case of the AS, the 2-layer model produces the WAG and the observed outflow of Mediterranean Water along the Moroccan continental slope [3]. Computed tides (2D depth-averaged model) have also been compared with observations in the AS and in the GoC. Both set of data are in good agreement.
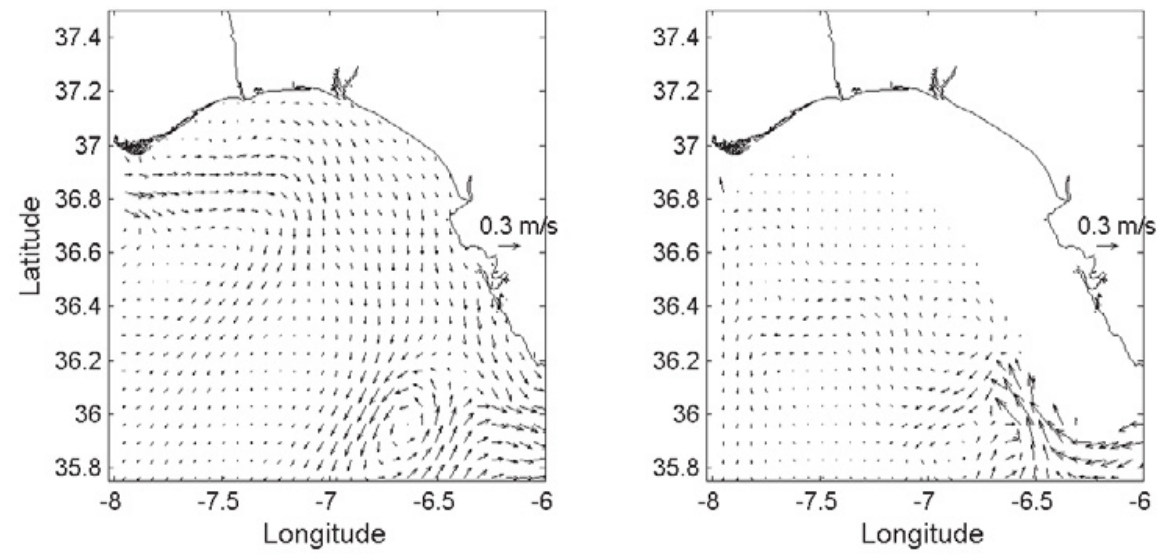

Figure 2. Residual circulation in the GoC at the surface (left) and at $190 \mathrm{~m}$ depth (right).
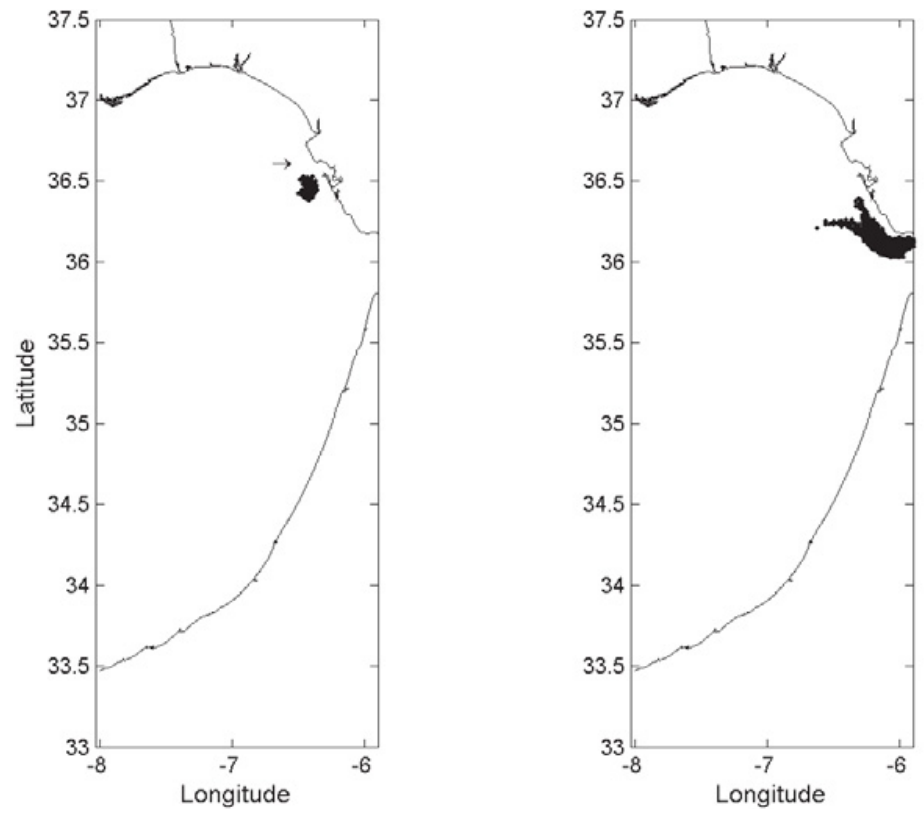

Figure 3. Positions of particles 2 (left) and 5 (right) days after an instantaneous radioactive spill occurring at the arrow position.

Some hypothetical accidents have been simulated to show the kind of results which may be obtained from the Lagrangian models. The first numerical experiment consisted of a radioactive spill in front of 
the Bay of Cadiz (arrow in Fig. 3). The spill was an instantaneous release at the surface on January 1, 2008 at 0:00 hours (this date and time was taken just as an example) and with no wind. Snapshots showing the position of particles 2 and 5 days after the accident may be seen in Fig. 3. These snapshots are projections of the three-dimensional particle positions on the $x y$ (horizontal) plain. The radioactive patch is transported towards the Strait of Gibraltar by the residual current, although a fraction is directed to the west by the gyre existing in front of the Strait (see Fig. 2).

a
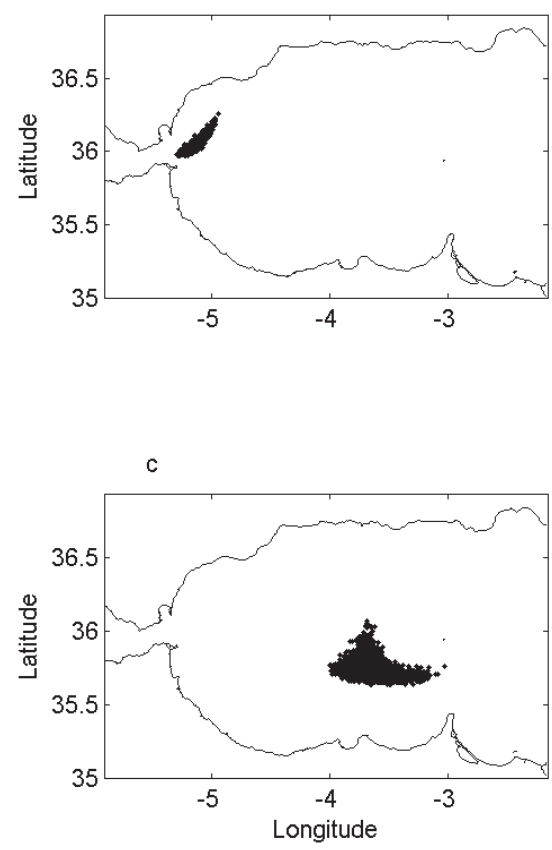

b

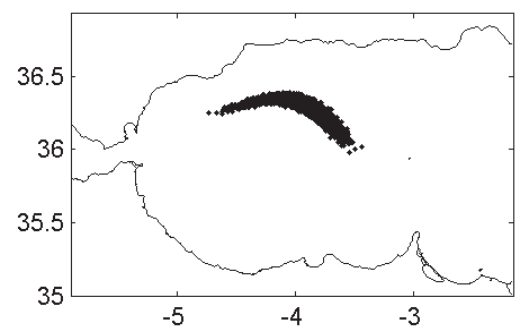

d

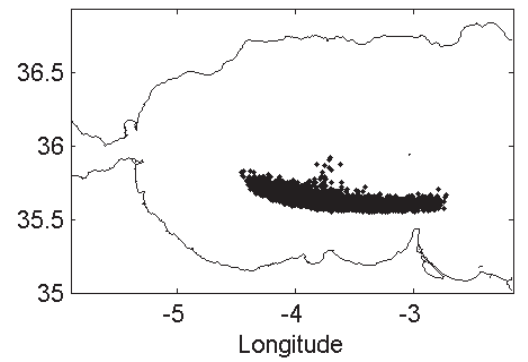

Figure 4. Position of particles 2.5 (a), 12,5 (b), 22,5 (c) and 30 (d) days after a continuous release in the center of the Strait of Gibraltar.

An example obtained with the AS model is presented in Fig. 4. In this case a continuous release of $48 \mathrm{~h}$ duration has been simulated. The accident was supposed to occur in the center of the Strait of Gibraltar, at the surface, and under calm wind. The total release was $1 \mathrm{TBq}$. Date and time were the same as in the previous example. It may be seen that the contamination patch moves along the Spanish coast and turns to the south because of the presence of the WAG. Most of the radionuclides remain trapped in the gyre, thus moving to the west again, and some of them are transported to the east close to the Moroccan coast. As a final example of result, radionuclide concentrations 30 days after the release are shown in Fig. 5.

\section{CONCLUSIONS}

Two rapid-response particle-tracking dispersion models covering the whole Andalusian coast have been described. These models are useful for radioactive contamination assessments after accidental or deliberate releases in the marine environment. The two models follow the same scheme: tides are obtained from a 2D depth averaged model and the residual circulation is obtained from a specific model suitable for the specific oceanographic conditions in each domain: a 2-layer model is used for the Alborán Sea and a 3D baroclinic model is applied in the Gulf of Cádiz. All the hydrodynamic calculations are carried out in advance and results are stored in files which are later read by the dispersion 
codes. Dispersion is simulated using a particle-tracking approach: a release is simulated by a number of discrete particles whose paths are followed in time. Turbulent diffusion is simulated by a radom-walk method and a stochastic approach is used to simulate radioactive decay. Radionuclide concentration at the desired time is obtained from the density of particles per water volume unit. Two accidents occurring in the GoC and in the AS have been simulated to provide some examples of results.
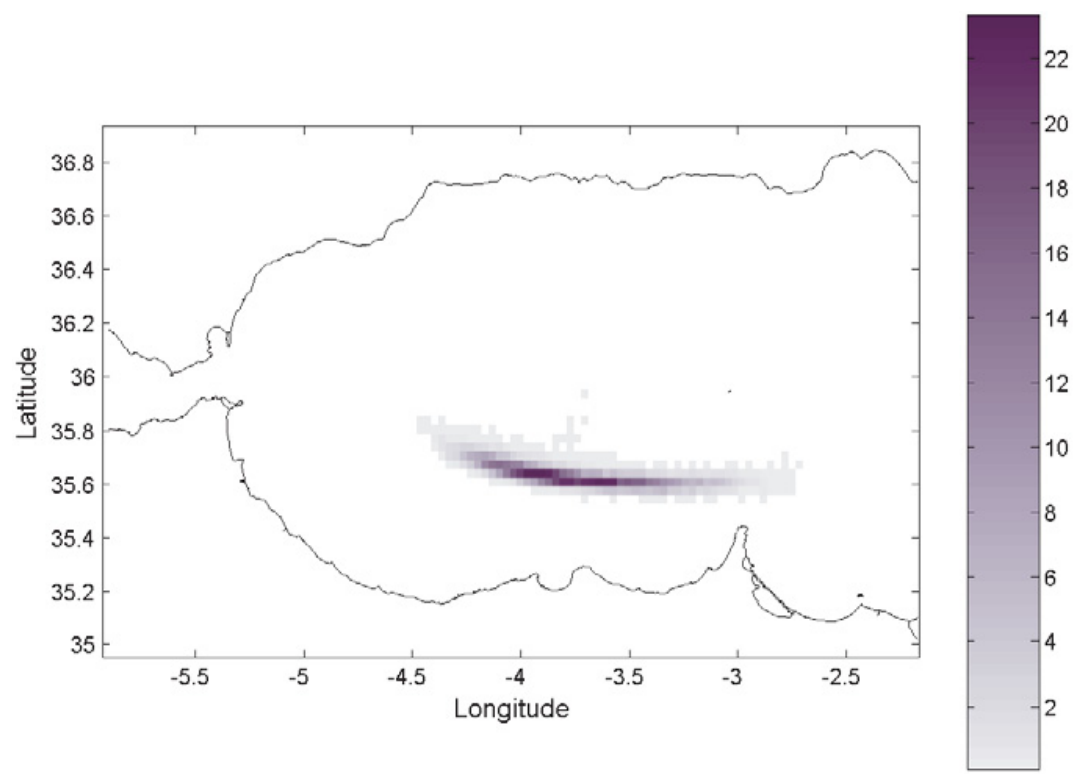

Figure 5. Computed surface concentration $\left(\mathrm{Bq} / \mathrm{m}^{3}\right) 30$ days after the accident in Fig. 4.

\section{Acknowledgments}

R. Periañez is indebted to the Spanish Ministerio de Educación y Ciencia for a fellowship to stay during three months in the University of Wales, where part of this work was carried out.

\section{References}

[1] Periáñez, R. J Environ Radioact 90 (2006), 48-67.

[2] Periáñez, R. Modelling the Dispersion of Radionuclides in the Marine Environment. SpringerVerlag, Heidelberg, 2005.

[3] Periáñez, R. J Environ Radioact 99 (2008), 694-715.

[4] Kowalick, Z. and Murty, T.S. Numerical Modelling of Ocean Dynamics. World Scientific, Singapore, 1993. 